\title{
Un método rápido y no-destructivo para medir la permeabilidad al aire del hormigón
}

Fecha de recepción: 2- VII-97

Fecha de aceptación: 4-IX-98
R. J. TORRENT

"Holderbank" Management \& Consulting Ltd.

SUIZA

\section{RESUMEN}

Se presenta un nuevo dispositivo de ensayo no destructivo, diseñado para la medición "in situ" de la permeabilidad al aire del recubrimiento de hormigón. Se basa en crear vacio dentro de una celda colocada sobre la superficie del mismo y en medir la velocidad con que la presión retorna al valor atmosférico.

Aspectos característicos del método son: una celda con doble cámara y un regulador que equilibra la presión en la cámara central (de medición) y en la cámara exterior (anillo de guarda). Esto asegura un flujo unidireccional de aire hacia la cámara central, eliminando todo flujo espúreo transversal. Los datos de la medición son procesados por un microprocesador que indica el valor del coeficiente de permeabilidad al finalizar el ensayo, que dura de 2 a 12 minutos.

Se presentan resultados obtenidos con esta técnica sobre recubrimientos de distinta calidad, que muestran una excelente correlación con los valores obtenidos sobre testigos mediante el método del Cembureau para permeabilidad al oxigeno.

\section{SUMMARY}

New device for non-destructive test. It is designed for "in situ" measurement of the impermeability to air of a concrete coating. The system is based on creating a vacuum inside a cell placed on its surface, and to measure how long does it take for the presure to be back to the atmospheric value.

The characteristic aspects of the method are: a cell with an inward chamber and a regulator to keep the pressure in balance inside this central chamber (measurement) and in the surrounding chamber (preservation ring). This ensures a single air flow direction towards the central chamber, avoiding any spurious transversal flow. The data of the measurement are processed by a microprocessor that indicates the permeability coefficient value at the end of the test which lasts 2 to 12 minutes.

Results on coatings of different quality obtained through this technique are presented, which show an excellent correlation with values obtained on specimens using Cembureau method for permeability to oxigen imperviousness.

\section{INTRODUCCIÓN}

Los serios problemas de durabilidad que se encuentran en muchas estructuras de hormigón, asociados, principalmente, a la depasivación y corrosión de las armaduras, al ataque químico p.ej. por sulfatos y a los ciclos de congelación y deshielo, han puesto en tela de juicio los enfoques tradicionales para la especificación y control del hormigón.
En tanto que la capacidad portante de un elemento estructural es la resultante de su comportamiento integral, su durabilidad frente a acciones agresivas del medio ambiente depende, básicamente, del desempeño protector de una capa superficial relativamente delgada $(20-50 \mathrm{~mm})$, como se ilustra en la Figura 1. Esta capa debe proteger a las armaduras contra la corrosión inducida por la carbonataciön o por ingreso de cloruros, y es también la más afectada 


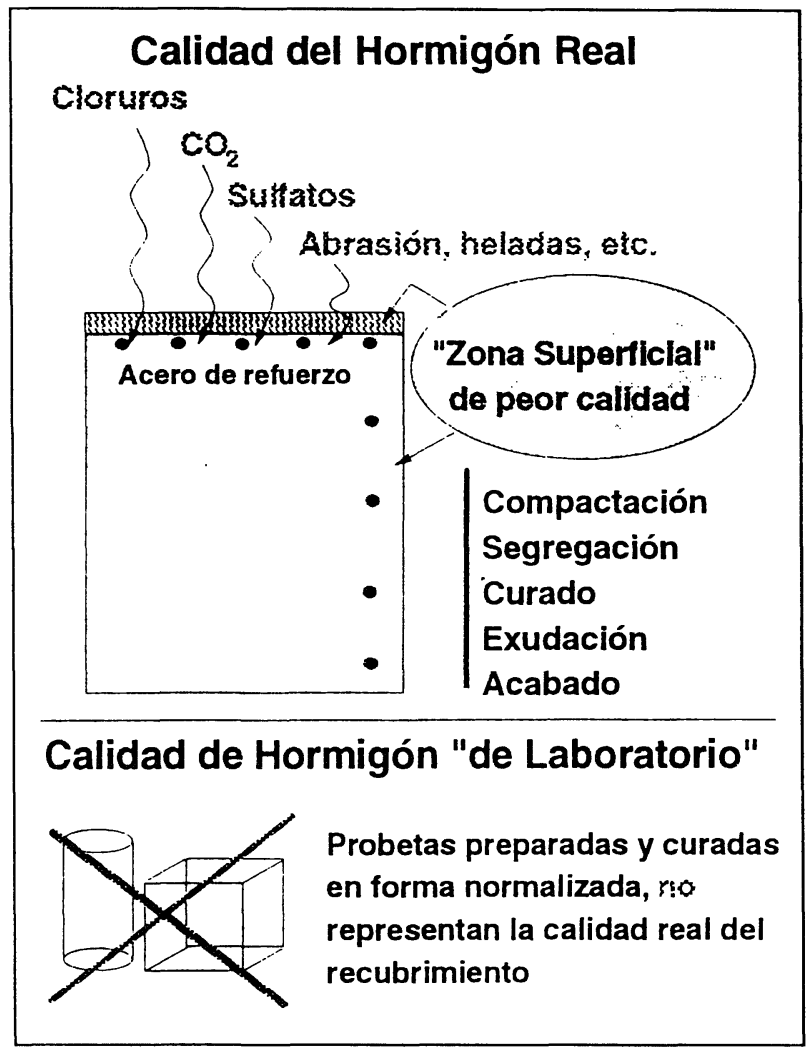

Figura 1.- Concepto de Hormigón Superficial (Recubrimiento).

por ataques químicos, congelación, etc. Lamentablemente, por ser la zona de más difícil compactación o por recibir las tareas de acabado y por ser la más expuesta a las consecuencias del mal hábito de no curar las estructuras, esta vital capa superficial suele ser la de peor calidad en el elemento estructural.

Actualmente, los criterios de aceptación del hormigón endurecido se basan, casi exclusivamente, en resultados de ensayos de probetas moldeadas, especialmente la resistencia a compresión. Está claro que esos resultados nunca pueden representar la calidad de la capa superficial, porque evalúan el comportamiento global de las probetas las cuales, además, se preparan y curan de una manera totalmente diferente de las condiciones reales en la estructura. Así, la calidad real de esa capa vital es ignorada lo que explica, al menos parcialmente, el desempeño insatisfactorio de muchas estructuras, desde el punto de vista de su durabilidad. La noción del hormigón de recubrimiento, que posee composición y propiedades diferentes a las del que se encuentra en el núcleo de las estructuras es relativamente nueva [1-4] aunque ya ha sido incorporada al Código Modelo CEB-FIP [5], que expresaba, en 1990 (traducción del inglés del autor):

"No existe un método de aceptación general para caracterizar la estructura de poros del hormigón y relacionarla con su durabilidad. Sin embargo, diversas investigaciones han indicado que la permeabilidad del hormigón, al aire o al agua, es una excelente medida de su resistencia al ingreso de medios agresivos en el estado gaseoso o líquido y asi constituye una medida de la durabilidad potencial de un hormígón dado."

"Actualmente no existen métodos de aceptación general para una determinación rápida de la permeabilidad del hormigón ni de valores límites de la permeabilidad del hormigón expuesto a diversas condiciones ambientales. Sín embargo, es probable que se disponga de tales métodos en el futuro, lo que permitirá clasificar al hormígón en base a su permeabilidad. Entonces podrán postularse requisitos para la permeabilidad del hormigón; ellos dependerán de las condiciones de exposición a que está sometida la estructura."

"Aunque el hormigón de mayor resistencia será, en la mayoria de los casos, más durable que el hormígón de menor resístencia, la resistencia a compresión 'per se' no es una medida completa de la durabilidad, porque ésta depende, principalmente, de las propiedades de las capas superficiales de un elemento de hormigón, las que ejercen sólo una influencia limitada sobre la resistencia a compresión. “

La calidad del recubrimiento es el resultado, por un lado, de la dosificación de la mezcla elegida y,-por el otro, del cuidado con el que el hormigón ha sido procesado (colocado, compactado, acabado, curado, etc.). Resulta obvio entonces que los ensayos sobre probetas moldeadas, que son procesadas de una manera completamente diferente a los elementos estructurales, no darán jamás una imagen realista y representativa de la calidad real del recubrimiento. Por lo tanto, la única posibilidad es medirla directamente en la estructura, preferentemente "in situ" con métodos nodestructivos o sino sobre testigos extraídos de ella. Está claro que habremos dado un paso muy importante cuando seamos capaces de especificar y controlar la calidad (y espesor) del recubrimiento, ya que ello permitirá asegurar una mayor durabilidad frente a las condiciones de exposición a que estará sometida la estructura.

El objeto de este trabajo es presentar una nueva técnica, no destructiva, que permite una determinación rápida y precisa del coeficiente de permeabilidad al aire del recubrimiento de hormigón. El método también puede ser aplicado en el laboratorio.

\section{DESCRIPCIÓN DEL MÉTODO}

\subsection{Descripción del aparato y de su operación.}

El equipo es una versión mejorada del descrito en [6]. La disposición del instrumental del aparato se esquematiza en la Figura 2. Sus dos características distintivas son: 
a) una celda con doble cámara, basada en el principio del anillo de guarda. Consiste en una cámara interna i y una cámara externa $\mathrm{e}$.

b) un regulador de presión a membrana, cuya función es mantener a ambas cámaras siempre a la misma presión $\left(P_{i}=P_{e}\right)$.

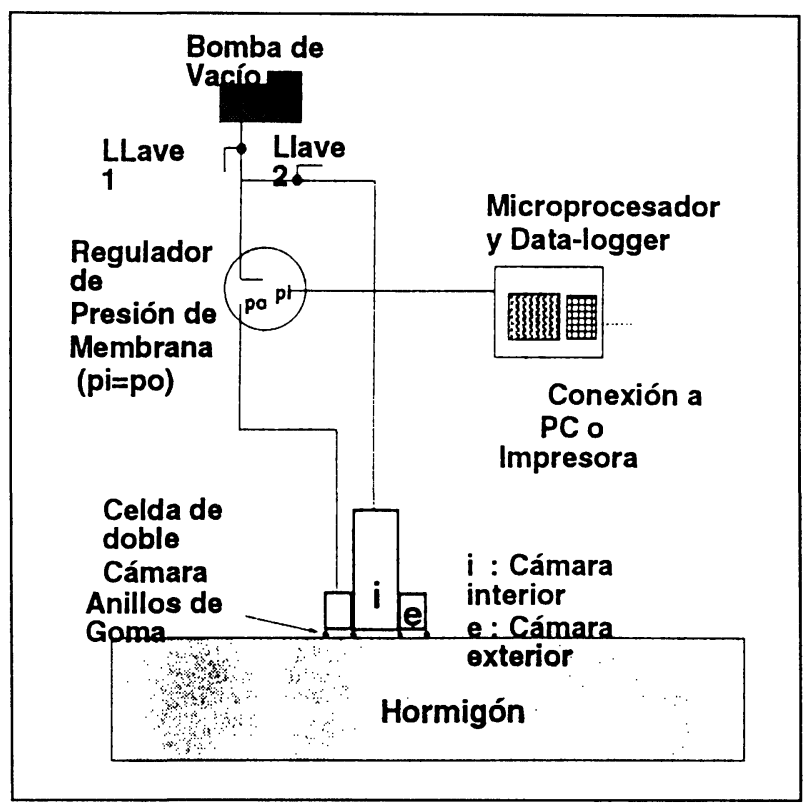

Figura 2.- Esquema del Permeabilímetro de Hormigón.

La operación del aparato es como sigue: con las llaves 1 y 2 abiertas, se crea vacío en ambas cámaras mediante la bomba. Luego de 1 minuto de crear vacío se cierra la llave 2, momento a partir del cual la bomba sólo puede actuar (cuando se lo permite el regulador) sobre la cámara externa, de manera que equilibra, en todo momento, la presión en ambas cámaras. De este modo, todo exceso de aire que ingrese lateralmente en la cámara externa será evacuado. Así se logra que el flujo de aire hacia la cámara central sea básicamente unidireccional y no afectado por el ingreso espúreo de aire, sea por un deficiente sellado de la cámara externa o a través de la más permeable 'piel'superficial (ver Figura 3).

La evolución de la presión $\mathrm{P}_{\mathrm{i}}$ se mide con un sensor de presión comandado por un microprocesador que tiene integrado un cronómetro. El microprocesador almacena la información y efectúa los cálculos para mostrar automáticamente, al final del ensayo, el valor del coeficiente de permeabilidad al aire $\mathrm{kT}\left(\mathrm{m}^{2}\right)$. El fin del ensayo acontece cuando la elevación de la presión en la cámara interna $P_{i}$, alcanza 20 mbar o, en el caso de hormigones muy impermeables cuando han transcurrido 12 minutos desde el comienzo del ensayo. Así, dependiendo de la permeabilidad del hormigón, el ensayo puede durar de 2 a 12 minutos. El microprocesador es capaz de almacenar los

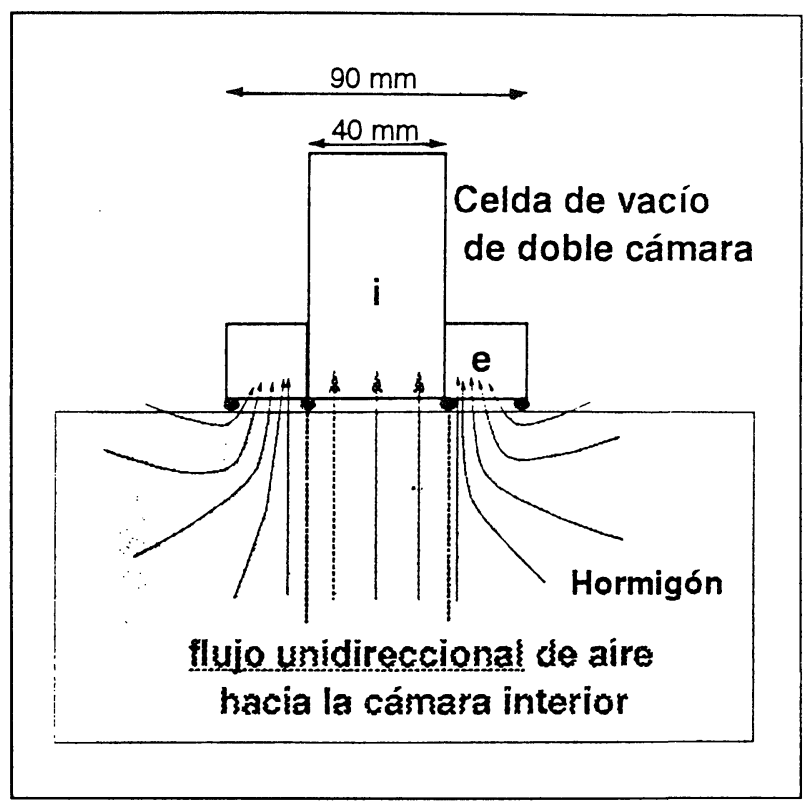

Figura 3.- Detalle del flujo de aire hacia la celda.

datos de unos 150 ensayos, y la información almacenada puede ser impresa o transferida a una PC para su posterior análisis.

La función de la llave 1 es restablecer el sistema para un nuevo ensayo, llenándolo con aire a la presión atmosférica.

\subsection{Cálculo del coeficiente de permeabilidad}

La evolución de la presión en la cámara interna presenta una forma como se indica en la Figura 4, para tres calidades de recubrimiento.

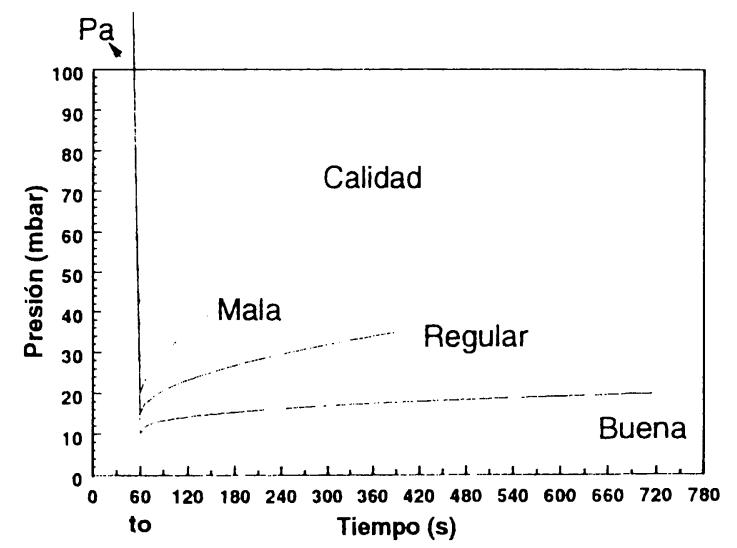

Figura 4.- Evolución de la presión en la cámara interna 
En ella pueden distinguirse dos etapas:

a) etapa de bombeo, entre 0 y to $=60 \mathrm{~s}$, caracterizada por una reducción de la presión $\mathrm{P}$ desde $\mathrm{Pa}$ hasta un valor menor de 50 mbar. Esta etapa finaliza al cerrarse la llave 2.

b) etapa de llenado, a partir de to, caracterizada por un incremento en $\mathrm{P}$ debido al aire que fluye a través del recubrimiento y que entra en la cámara interna. El aumento de presión será más rápido cuanto más permeable sea el recubrimiento ensayado. El ensayo termina cuando el incremento de presión alcanza unos 20 mbar o cuando hayan transcurrido $12 \mathrm{~min}(720 \mathrm{~s})$ desde el comienzo del ensayo.

Dado que en este método la geometría del problema está bien definida, mediante un modelo teórico es posible calcular el coeficiente de permeabilidad, tal como se describe en [7]. La clave del cálculo es la determinación del único elemento no definido directamente por el ensayo: la profundidad afectada $(L)$, en la cual, todavía, los poros están a la presión atmosférica.

Como se detalla en [7], el coeficiente de permeabilidad al aire viene calculado como:

$$
k T=\left(V_{c} / A\right)^{2} \cdot \frac{\mu}{2 \varepsilon P_{a}}\left[\frac{\ln \left(\frac{P_{a}+P}{P_{a}-P} \cdot \frac{P_{a}-P_{0}}{P_{a}+P_{0}}\right)}{\sqrt{t}-\sqrt{t_{0}}}\right]^{2}
$$

donde:

$\mathrm{kT}=$ coeficiente de permeabilidad al aire $\left(\mathrm{m}^{2}\right)$

$\mu=$ viscosidad dinámica del aire $\left(\mathrm{N} \cdot \mathrm{s} / \mathrm{m}^{2}\right)$

$\mathrm{V}_{\mathrm{c}}=$ capacidad de la cámara central más los elementos auxiliares en los que penetra el aire durante la etapa de llenado $\left(\mathrm{m}^{3}\right)$

$\varepsilon=$ porosidad vacía del hormigón $\left(\mathrm{m}^{3} / \mathrm{m}^{3}\right)$

$\mathrm{A}_{\mathrm{c}}=$ sección del 'cilindro' por el que el aire fluye dentro de la cámara central $\left(\mathrm{m}^{2}\right)$

$\mathrm{P}_{\mathrm{a}}=$ presión atmosférica $\left(\mathrm{N} / \mathrm{m}^{2}\right)$

$\mathrm{t}=$ tiempo $(\mathrm{s})$

$\mathrm{P}=$ presión en la cámara central en el instante $\mathrm{t}\left(\mathrm{N} / \mathrm{m}^{2}\right)$

$\mathrm{P}_{\mathrm{o}}=$ presión en la cámara central en el instante to $=60 \mathrm{~s}$ $\left(\mathrm{N} / \mathrm{m}^{2}\right)$

El conocimiento de $\mathrm{kT}$ permite estimar la profundidad L de hormigón afectada por el ensayo, mediante:

$$
L-\sqrt{\frac{2 \cdot k T \cdot P_{a}}{\varepsilon \mu} \cdot \sqrt{t}}
$$

$L$ es la distancia $(m)$ desde la superficie de hormigón a la cual los poros se encuentran aún a la presión atmosférica original y su valor es también mostrado en la pantalla del microprocesador al final del ensayo.

\section{RESULTADOS EXPERMMNTALES}

La técnica de ensayo propuesta fue aplicada a cuatro pares de cubos de hormigón de $1 \mathrm{~m}$ de arista, preparados independientemente en la Escuela Politécnica Federal de Zürich (ETH). Durante las mediciones se ignoraba la identidad de cada cubo.

Cada par de cubos correspondía a una composición diferente; uno de ellos no recibió ningún curado húmedo (Cubo A) y el otro fue curado en cámara húmeda durante 7 días (Cubo B).

A la edad de 28 días se efectuaron 10 lecturas de $\mathrm{kT}$ sobre cada cubo, 5 en cada una de dos caras verticales opuestas, promediándoselas.

La Figura 5 muestra los resultados obtenidos con el permeabilímetro, pudiéndose ver que el mismo detecta muy bien los cambios en permeabilidad, ya sean debidos a la diferente resistencia del hormigón o, por otro lado, al distinto curado. Vale la pena destacar que la falta de curado equivale, desde el punto de vista de la durabilidad, a desperdiciar $100 \mathrm{kgf} / \mathrm{cm}^{2}$ de resistencia (las resistencias indicadas corresponden a cilindros de $150 \times 300 \mathrm{~mm}$ con 28 días de curado húmedo). Las clases indicadas a la derecha del gráfico se explican más abajo.

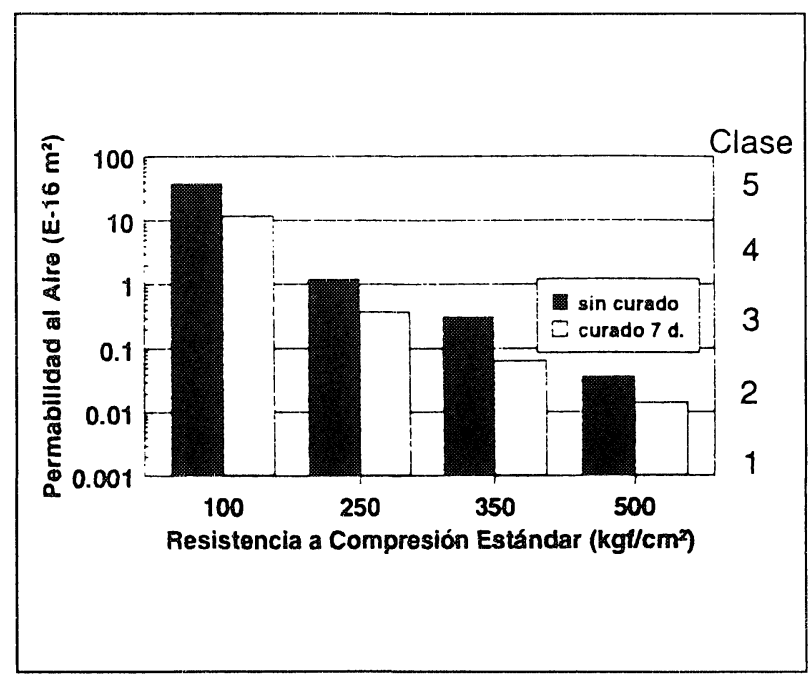

Figura 5.- Influencia de la Resistencia y el Curado sobre la Permeabilidad al Aire kT. 
Luego de efectuado el ensayo no-destructivo, se extrajeron testigos de $\phi=150 \mathrm{~mm}$ de los mismos cubos, los cuales se cortaron para obtener discos de $50 \mathrm{~mm}$ de espesor. Estos testigos fueron secados durante seis días en estufa a $50^{\circ} \mathrm{C}$ y luego enfriados a $20^{\circ} \mathrm{C}$ durante un día en un desecador. Entonces fueron sometidos al ensayo de permeabilidad al oxígeno según el método del Cembureau [8]. La comparación entre las dos mediciones de permeabilidad a los gases se presenta en la Figura 6, donde puede verse que el coeficiente de permeabilidad determinado por el método se correlaciona muy bien con el método del Cembureau y que puede tomarse como método de referencia. Esto confirma experiencias anteriores con versiones previas del equipo $[6,7]$.

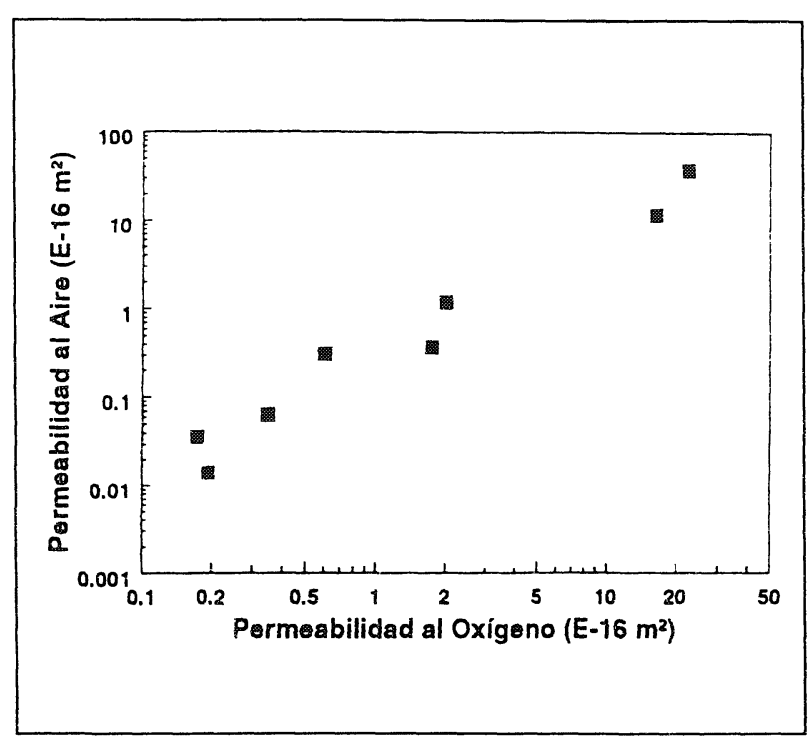

Figura 6.- Correlación entre el Método Propuesto y el Método de Permeabilidad al Oxígeno del Cembureau.

\section{CLASIFICACIÓN DE LA CALIDAD DEL RECUBRIMIENTO}

En base a numerosos resultados obtenidos con el método, tanto en el laboratorio como en obra, se propone una clasificación de la calidad del recubrimiento en función de su permeabilidad al aire, medida a una edad de 1 a 3 meses (hormigones 'jóvenes'). La Clase 1 corresponde a hormigones de alta resistencia ( $>60 \mathrm{MPa})$, bien compactados y curados, en tanto que la clase 5 corresponde a hormigones de baja resistencia (>15 MPa) mal curados.

Esto puede confirmarse viendo los resultados de la Figura 5 .

Los límites indicados corresponden al caso en el cual se efectua la medición sobre hormigón relativamente seco. Puede suponerse que después de una semana, o

\begin{tabular}{|c|l|c|}
\hline Clase $\mathrm{N}^{\circ}$ & Descripción & $\mathrm{kT}\left(10^{-16} \mathrm{~m}^{2}\right)$ \\
\hline 1 & Excelente & $<0.01$ \\
\hline 2 & Muy Buena & $0.01-0.1$ \\
\hline 3 & Normal & $0.1-1$ \\
\hline 4 & Pobre & $1-10$ \\
\hline 5 & Muy Mala & $>10$ \\
\hline
\end{tabular}

preferentemente dos, de no haber estado en contacto con agua (curado, lluvia, salpicaduras) el recubrimiento estará en condición, suficientemente seca, como para efectuar el ensayo de permeabilidad al aire. En caso contrario, el mismo debe complementarse con uno de resistividad eléctrica para compensar la influencia de la humedad sobre la permeabilidad. En este último caso se establece la Clase a la que pertenece un recubrimiento mediante el uso del nomograma mostrado en la Figura 7.

Una idea del sentido del nomograma puede obtenerse analizando los puntos $\mathrm{a}, \mathrm{b}, \mathrm{c}$ y d indicados en el mismo. Todos los puntos corresponden a una medida muy baja de permeabilidad. Los puntos c y d pertenecen realmente a la Clase 1 (Excelente) pues su resistividad eléctrica es alta, lo que es compatible con un hormigón de alta calidad. El punto $\mathrm{b} \mathrm{y}$, más aún, el a, debido a su baja resistividad eléctrica, corresponden a hormigones de calidades inferiores, pero que están húmedos (baja resistividad y permeabilidad).

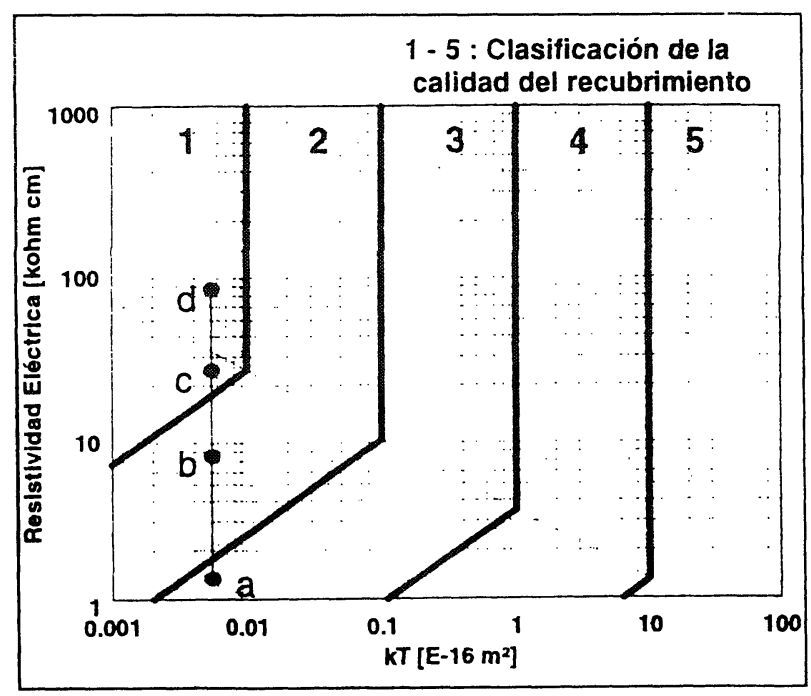

Figura 7.- Nomograma para Clasificar el Recubrimiento en base a la Permeabilidad al Aire y la Resistividad Eléctrica.

\section{CONCLUSIONES}

Se presenta una nueva técnica para la evaluación "in situ" de la permeabilidad al aire del recubrimiento. Dicha técnica 
resulta superior a las existentes, gracias a las siguientes ventajas:

. Es absolutamente no-destructiva

- Es rápida (2 a 12 min por determinación), precisa y repetible

. No queda afectada por el ingreso espúreo de aire, sea por insuficiente estanquidad de la celda o por 'corto-circuito' a lo largo de la 'piel' superficial más permeable.

- El flujo de aire es; básicamente, unidireccional y perpendicular a la superficie expuesta, o sea, en la misma dirección, aunque en sentido inverso que el transporte real de fluidos agresivos dentro del hormigón.

. La clara definición geométrica del flujo de aire permite calcular, en base a un modelo teórico, el coeficiente de permeabilidad al aire.

El mismo modelo permite estimar la profundidad de hormigón afectada por el ensayo.

Los resultados obtenidos con el nuevo método indican, no sólo una excelente correlación, sino también una muy buena aproximación al coeficiente de permeabilidad al oxígeno, medido por el método del Cembureau.

En casos donde las mediciones deban efectuarse sobre hormigón que no está suficientemente seco, el ensayo de permeabilidad al aire debe ser complementado por el de resistividad eléctrica.

Este método puede constituir un paso importante para la aplicación práctica de los conceptos expresados en el
Código Modelo CEB-FIP, o sea la especificación de la permeabilidad del recubrimiento para distintos grados de exposición y el control en obra de su cumplimiento.

\section{BIBLIOGRAFÍA}

(1) KREIJGER, P.C.: "The skin of concrete. Composition and properties”, Mater. \& Struct. 17(100) (1984) 275-283.

(2) NEWMAN, K.: "Labcrete, realcrete and hypocrete. Where we can expect the next major durability problems", ACI SP-1 00 (1 987), v.2, 1259-1283.

(3) MEYER, A.: "The importance of the surface iyesterday for the durability of concrete structures!", ACI SP-100 (1987), v.1, 49-61.

(4) TORRENT, R.J.: "La calidad del hormigón de recubrimiento, factor vital para la durabilidad de las estructuras", Jornadas Tecnol. AATH, Buenos Aires, 3031 de agosto de 1990.

(5) CEB-FIP MODELCODE 1990, Final Draft, Sectiond.5.3.: "Classification by Durability", CEB Bulletin d'information No 205, Lausanne, julio 1991.

(6) TORRENT, R.J.: “A two-chamber vacuum celi for measuring the coefficient of air-permeability of the concrete cover on site", Materials \& Structures, v. 25, n.150, julio 1992, pp. 358-365.

(7) TORRENT, R. and FRENZER, G.: "Methoden zurMessung und Beurteiiung der Kennwert des Ueberdeckungsbetons auf der Bausteile", Report No 516, Office Fédéral des Routes, Zürich, octubre 1995.

(8) KOLIEK, J.J.: "The determination of the permeability of concrete to oxygen by the Cembureau Method a recommendation", Mater. \& Struct., v.22(1989) 225-230. 